Absorbing boundary conditions for dynamical many-body quantum systems. Sølve Selstø and Simen Kvaal.

J. Phys. B: At. Mol. Opt. Phys. 43 (2010) 065004 (9pp)

doi:10.1088/0953-4075/43/6/065004

(C2010 IOP Publishing Ltd

Online at http://stacks.iop.org/JPhysB/43/065004

Access to the published version may require journal subscription.

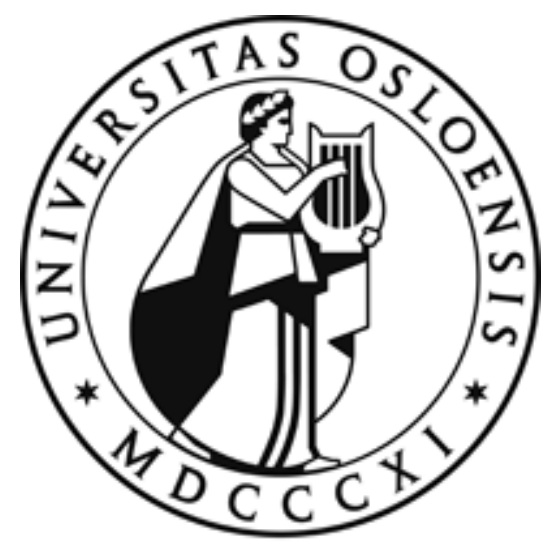


Absorbing boundary conditions for dynamical many-body quantum systems

This article has been downloaded from IOPscience. Please scroll down to see the full text article.

2010 J. Phys. B: At. Mol. Opt. Phys. 43065004

(http://iopscience.iop.org/0953-4075/43/6/065004)

The Table of Contents and more related content is available

Download details:

IP Address: 129.240.222.68

The article was downloaded on 15/03/2010 at 07:42

Please note that terms and conditions apply. 


\title{
Absorbing boundary conditions for dynamical many-body quantum systems
}

\author{
Sølve Selstø and Simen Kvaal \\ Centre of Mathematics for Applications, University of Oslo, N-0316 Oslo, Norway \\ E-mail: solve@cma.uio.no and simenkva@matnat.uio.no \\ Received 15 December 2009, in final form 3 February 2010 \\ Published 9 March 2010 \\ Online at stacks.iop.org/JPhysB/43/065004
}

\begin{abstract}
In numerical studies of the dynamics of unbound quantum mechanical systems, absorbing boundary conditions are frequently applied. Although this certainly provides a useful tool in facilitating the description of the system, its applications to systems consisting of more than one particle are problematic. This is due to the fact that all information about the system is lost upon the absorption of one particle; a formalism based solely on the Schrödinger equation is not able to describe the remainder of the system as particles are lost. Here we demonstrate how the dynamics of a quantum system with a given number of identical fermions may be described in a manner which allows for particle loss. A consistent formalism which incorporates the evolution of sub-systems with a reduced number of particles is constructed through the Lindblad equation. Specifically, the transition from an $N$-particle system to an $(N-1)$-particle system due to a complex absorbing potential is achieved by relating the Lindblad operators to annihilation operators. The method allows for a straight forward interpretation of how many constituent particles have left the system after interaction. We illustrate the formalism using one-dimensional two-particle model problems.
\end{abstract}

(Some figures in this article are in colour only in the electronic version)

\section{Introduction and basic theory}

Great effort has been invested in understanding the quantum mechanical behaviour of dynamical unbound systems involving several particles. Experimental advances allow us to study processes in which internal interactions between the constituent particles play a crucial role-in addition to dynamics induced by external perturbations. Examples of such processes may be photo-ionization of helium [1] and cascaded Auger processes following ionization of inner-core electrons in atoms [2]. Of course, in order to understand such processes, theoretical and numerical studies are required. Furthermore, the theoretical interest of such systems, be it within the context of solid state, molecular, atomic or nuclear physics, is spurred by the fact that they represent demanding tasks. One obvious challenge in describing unbound systems is that their spatial extension may become arbitrarily large. Moreover, even if one is able to represent the whole system within a finite space, extracting relevant information from the final wavefunction may be far from trivial.

The unbound quantum systems under study may often be thought of as having an interaction region of finite spatial extension and an asymptotic region where the unbound part of the system travels outwards. As this asymptotic behaviour often is well known, it may be desirable to describe only the dynamics of the part of the wavefunction belonging to the interaction region. In a numerical implementation this cannot be done simply by resorting to a representation of space that is smaller than the extension of the wavefunction as this leads to unphysical reflections at the boundary. However, it can be achieved by imposing absorbing boundary conditions, i.e. by demanding that the wavefunction vanishes as the particle approaches the edge of the numerical grid-preferably with as little reflection as possible, see [3, 4] or the recent review [5]. Such absorbers are frequently referred to as perfectly matched layers in the context of general wave equations [6].

When propagating a wave packet on a numerical grid, a common way to impose absorbing boundary conditions is by adding a complex absorbing potential (CAP) to the Hamiltonian of the system [7]. Complex absorbing potentials are widely used e.g. within molecular dynamics [8-10] and atomic physics $[11,12]$. Alternatively, this way of absorbing 
particles may be formulated as multiplying the wavefunction with a masking function at each time step [1].

In any case the resulting effective Hamiltonian acquires an anti-Hermitian part

$$
H_{\text {eff }} \equiv H-\mathrm{i} \Gamma,
$$

where both $H$ and $\Gamma$ are Hermitian and $\Gamma$ is positive semidefinite $(\Gamma \geqslant 0)$.

The wavefunction $|\Psi(t)\rangle$ of the system obeys the Schrödinger equation

$$
\mathrm{i} \hbar \frac{\mathrm{d}}{\mathrm{d} t}|\Psi(t)\rangle=H_{\mathrm{eff}}|\Psi(t)\rangle
$$

A density operator $\rho(t)$ correspondingly obeys the von Neumann equation

$\mathrm{i} \hbar \frac{\mathrm{d}}{\mathrm{d} t} \rho(t)=H_{\mathrm{eff}} \rho(t)-\rho(t) H_{\mathrm{eff}}^{\dagger}=[H, \rho(t)]-\mathrm{i}\{\Gamma, \rho(t)\}$.

It is easy to see that the evolution is non-unitary, namely

$$
\frac{\mathrm{d}}{\mathrm{d} t} \operatorname{Tr}[\rho(t)]=-\frac{2}{\hbar} \operatorname{Tr}[\Gamma \rho(t)] \leqslant 0,
$$

so that probability is 'lost' if the density matrix overlaps with the anti-Hermitian part of the effective Hamiltonian.

Although methods involving non-Hermitian effective Hamiltonians may come in very handy in reducing the complexity in describing potentially unbound systems, there is one major problem when the system contains more than one particle: as one particle leaves the rest of the system and is subsequently absorbed, the entire wavefunction is lost. This is obvious since the wavefunction is normalized to the probability of finding all particles within the space defined by the numerical implementation. In other words, if an initial $\mathrm{N}$-particle system gradually 'loses' one particle through some non-Hermitian 'interaction', the corresponding wavefunction $\left|\Psi_{N}(t)\right\rangle$ gradually goes to zero-not to some wavefunction corresponding to $(N-1)$ particles.

As it is desirable to be able to describe dynamics where several particles are lost one by one, one may try and construct a formalism where an $(N-1)$-particle wavefunction $\left|\Psi_{N-1}(t)\right\rangle$ is created as one particle is lost, and where this wavefunction may be propagated using the corresponding Schrödinger equation including some source term. Once able to do this, the extension to $(N-2)$ particles etc follows by induction. However, as it turns out, such a construction is not possible due to the fact that the process of losing particles in this way is irreversible: as a particle is absorbed, information is irretrievably lost. Hence, a Markovian master equation should be a more suitable starting point than a pure state approach. One such equation is the Lindblad equation:

$$
\mathrm{i} \hbar \frac{\mathrm{d}}{\mathrm{d} t} \rho=[H, \rho]-\mathscr{D}(\rho)
$$

with the so-called Lindbladian $\mathscr{D}(\rho)$ given by the generic expression [13]

$$
\mathscr{D}(\rho)=\mathrm{i} \sum_{m, n} \gamma_{m, n}\left(A_{m}^{\dagger} A_{n} \rho+\rho A_{m}^{\dagger} A_{n}-2 A_{n} \rho A_{m}^{\dagger}\right)
$$

Here, $H$ is the Hamiltonian governing the unitary part of the evolution. The operators $A_{n}$ are referred to as Lindblad operators. The matrix formed by the coefficients $\gamma_{m, n}$ is required to be positive semi-definite. In a diagonal representation, the Lindbladian simplifies to

$$
\mathscr{D}(\rho)=\mathrm{i} \sum_{n}\left(A_{n}^{\dagger} A_{n} \rho+\rho A_{n}^{\dagger} A_{n}-2 A_{n} \rho A_{n}^{\dagger}\right) .
$$

Equation (5) is commonly used to describe energy dissipation to the environment [14]. It is also frequently encountered in the context of quantum optics [15]. For instance, when describing the composite system consisting of an atom in a quantized radiation field, the description of the reduced density matrix obtained by fixing the number of photons to $n$ and tracing out the field part of the density matrix of the composite system

$$
\rho^{(n)}=\operatorname{Tr}_{F}\left[P_{F}^{(n)} \rho\right],
$$

where $P_{F}^{(n)}$ projects onto the $n$-photon subspace and index $F$ refers to the photon field, may be described by the Lindblad equation [16].

The Lindblad equation is often constructed from the Born-Markov equation by insisting that the system density matrix $\rho$ (or $\rho^{(n)}$ in the above example) be positive semidefinite at all times. However, it has been proven by Gorini, Kossakowki and Sudarshan [17], and by Lindblad [18] that equation (5) with the Lindbladian (6) is the most general formulation of a Markovian process that ensures positivity and conservation of the trace of the density matrix. As the processes we wish to describe should fulfil precisely these criteria, and since in the present case there is no reference to any external system with which the particles interact, the Lindblad equation seems to be the proper starting point for our purpose.

It has recently been demonstrated that the spontaneous decay of unstable particles may also be described within this formalism [19, 20]. In [19] a master equation of Lindblad form is obtained for a Hilbert space consisting of unstable particle states and vacuum-however, without any dynamical degrees of freedom. The decay rates of the unstable particles are introduced as parameters. Similarly, in [20] decay from one system to another one consisting of its decay products is described by introducing a single Lindblad operator. In these works it is demonstrated that one may describe decoherence along with decay in this context.

In the present work these ideas have been used to generalize the standard one-particle absorbing boundary technique to an $N$-body setting, and it is demonstrated that this is indeed the natural way to do this. Alternatively, in a more general context, this formalism allows the study of a system which gradually loses particles to some environment due to the non-Hermitian part of $H_{\text {eff }}$.

We comment that the absorber should not affect the dynamics in its interior. Its overlap with any confining potential must be very small and it should induce as little reflection and transmission as possible. This is readily seen to be true for any implementation of absorbing boundary conditions. The situation is slightly more complicated for many-particle systems, however, than for one-particle systems. One must additionally assume that the absorber is placed sufficiently far away from the confining potential so that the interaction between outgoing particles and the remaining ones 
can be neglected. This may effectively force the CAP further away from the confinement region.

In the following section, section 2, the formalism will be described for identical fermions exposed to a complex absorbing potential. In section 3 , two examples featuring two identical fermions in one dimension are given. Finally, in section 4 , conclusions are drawn and a few future perspectives are outlined.

\section{Fock space description}

The natural setting for describing a system of a variable number of fermions is Fock space, the direct sum of all $n$-fermion spaces $\mathscr{H}_{n}$. As we wish to describe at most $N$ particles, it suffices to consider

$$
\mathscr{H}=\bigoplus_{n=0}^{N} \mathscr{H}_{n}
$$

An arbitrary $n$-fermion state $|\Psi\rangle \in \mathscr{H}_{n}$ can be written as

$$
|\Psi\rangle=\frac{1}{n !} \int \mathrm{d} x^{n} \Psi\left(x_{1} \cdots x_{n}\right) \psi^{\dagger}\left(x_{1}\right) \cdots \psi^{\dagger}\left(x_{n}\right)|-\rangle,
$$

where the field operator $\psi^{\dagger}(x)$ creates a particle at position $x$. The field operators obey the usual anti-commutator relations

$$
\left\{\boldsymbol{\psi}(x), \boldsymbol{\psi}\left(x^{\prime}\right)\right\}=0, \quad\left\{\boldsymbol{\psi}(x), \boldsymbol{\psi}^{\dagger}\left(x^{\prime}\right)\right\}=\delta\left(x-x^{\prime}\right) .
$$

Here, $x$ may denote all degrees of freedom associated with a particle, including eigenspin. Moreover, $\Psi\left(x_{1} \cdots x_{n}\right)$ is the anti-symmetric local wavefunction of the $n$-particle system, and $|-\rangle \in \mathscr{H}_{0}$ is the vacuum state, containing zero particles.

A system of $n$ identical fermions interacting with at most two-body forces has the Hamiltonian

$$
H=\sum_{i=1}^{n} h\left(x_{i}\right)+\sum_{j<i}^{n} u\left(x_{i}, x_{j}\right) .
$$

Here, $h\left(x_{i}\right)$ (resp. $\left.u\left(x_{i}, x_{j}\right)\right)$ is a one-body (two-body) operator acting on the degrees of freedom associated with particle $i$ (and $j$ ). Using field operators, the Hamiltonian can be written as

$$
\begin{aligned}
H= & \int \mathrm{d} x \boldsymbol{\psi}^{\dagger}(x) h(x) \psi(x) \\
& +\frac{1}{2} \int \mathrm{d} x \mathrm{~d} x^{\prime} \boldsymbol{\psi}^{\dagger}(x) \boldsymbol{\psi}^{\dagger}\left(x^{\prime}\right) u\left(x, x^{\prime}\right) \psi\left(x^{\prime}\right) \psi(x),
\end{aligned}
$$

the point here being that $H$ given in this form is independent of the number of particles in the system. The exact expression is irrelevant at this point. The CAP, which is diagonal in $x$, is conveniently introduced as

$$
-\mathrm{i} \Gamma=-\mathrm{i} \int \mathrm{d} x \boldsymbol{\psi}^{\dagger}(x) \Gamma(x) \psi(x) .
$$

By comparing (5), (7) and (14) with the von Neumann equation (3), we see that the Lindblad operators, for a diagonal representation of the Lindbladian, must fulfil

$$
\Gamma=\int \mathrm{d} x A^{\dagger}(x) A(x)
$$

in order to reproduce the anti-Hermitian 'interaction' leading to absorption. Here we have allowed for an integral instead of a sum in (7). Furthermore, the last term of the Lindbladian,

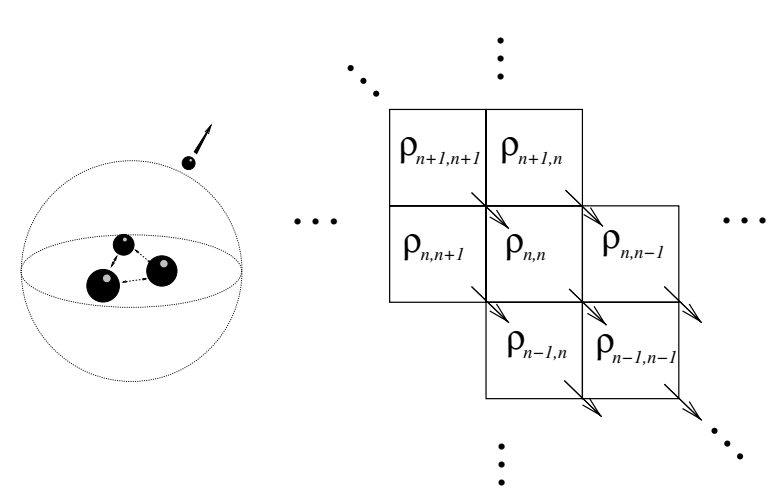

Figure 1. Illustration of how the various blocks of the density matrix are populated via the one above to the left only. As a consequence of this, when the density matrix is block diagonal, this diagonal structure is maintained, and losing a particle (left figure) corresponds to moving downwards along the block diagonal of $\rho$.

which is absent in the von Neumann equation, $-2 A_{n} \rho A_{n}^{\dagger} \rightarrow$ $-2 A(x) \rho A^{\dagger}(x)$, should map an $N$-particle system into an $(N-1)$-particle system. Hence, $A(x)$ should map $\mathscr{H}_{N}$ into $\mathscr{H}_{N-1}$, which means that the Lindblad operator $A(x)$ must be of the form

$$
A(x)=\int \mathrm{d} x a\left(x, x^{\prime}\right) \psi\left(x^{\prime}\right)
$$

The simplest choice that satisfies (15) is the diagonal form

$$
A(x) \equiv \sqrt{\Gamma(x)} \psi(x) .
$$

We will return to the justification of why this is the proper way to define the Lindblad operators shortly.

With (17), the Lindbladian (7) may immediately be written as

$$
\mathscr{D}(\rho)=\mathrm{i} \Gamma \rho+\mathrm{i} \rho \Gamma-2 \mathrm{i} \int \mathrm{d} x \Gamma(x) \psi(x) \rho \psi^{\dagger}(x),
$$

and the master equation (5) becomes

$\mathrm{i} \hbar \frac{\mathrm{d}}{\mathrm{d} t} \rho=[H, \rho]-\mathrm{i}\{\Gamma, \rho\}+2 \mathrm{i} \int \mathrm{d} x \Gamma(x) \psi(x) \rho \psi^{\dagger}(x)$.

This is our fundamental dynamical formulation of particle loss due to a CAP.

Let us consider the master equation (19) in some detail. We may partition the density matrix $\rho$ into blocks, namely

$$
\rho=\sum_{n=0}^{N} \sum_{m=0}^{N} \rho_{n, m},
$$

where $\rho_{n, m}=P_{n} \rho P_{m}$, with $P_{n}$ being the orthogonal projector onto $\mathscr{H}_{n}$. Each block evolves according to

$$
\begin{aligned}
& \mathrm{i} \hbar \frac{\mathrm{d}}{\mathrm{d} t} \rho_{m, n}=\left[H, \rho_{m, n}\right]-\mathrm{i}\left\{\Gamma, \rho_{m, n}\right\} \\
& \quad+2 \mathrm{i} \int \mathrm{d} x \Gamma(x) \psi(x) \rho_{m+1, n+1} \psi^{\dagger}(x)
\end{aligned}
$$

showing that the flow of $\rho_{n, m}$ depends on that of $\rho_{n+1, m+1}$, but not the other way around. This is illustrated in figure 1. Also, note that $\rho_{N, N}$ obeys the original von Neumann equation (3) as there are no couplings to other blocks in this case as particles do not enter the $N$-particle system. 
We now return to the question of whether definition (17) of the Lindblad operators, which obviously is the simplest one, is the only adequate one. Indeed, during the transition from an $N$-particle system to an $(N-1)$-particle system, only the unabsorbed part of the original system should be reproduced within the $(N-1)$-particle system, and this leads to the above choice. To see this, consider a somewhat idealized example consisting of two non-interacting particles. Since they do not interact, their wavefunction is given by a Slater determinant at all times, $\Psi=\left[\alpha\left(x_{1}, t\right) \beta\left(x_{2}, t\right)-\beta\left(x_{1}, t\right) \alpha\left(x_{2}, t\right)\right] / \sqrt{2}$. The state $\alpha$ does not overlap with the absorber at any time, i.e.

$$
\Gamma(x) \alpha(x, t)=0, \quad \text { for all } x \text { and } t,
$$

and $\beta$ corresponds to an unbound particle travelling outwards. We suppose that as $t$ approaches infinity, the particle in the state $\beta$ is completely absorbed, and our numerical representation of the final system should converge towards the pure one-particle state $\alpha$.

Indeed, the evolution dictated by (21) follows this pattern. The block of the density matrix corresponding to the twoparticle system, $\rho_{2,2}$, remains a pure state-albeit with decreasing norm as $\beta$ is absorbed-and the evolution of the one-particle block $\rho_{1,1}$ simplifies due to (22) to

$$
\mathrm{i} \hbar \dot{\rho}_{1,1}=\left[h, \rho_{1,1}\right]+2 \mathrm{i} \int \Gamma(x)|\beta(x ; t)|^{2} \mathrm{~d} x|\alpha\rangle\langle\alpha| .
$$

Hence, the one-particle part of the system is always proportional to a pure $\alpha$-state, and, since the trace of the entire system is conserved, this simply integrates to $\rho_{1,1}(t \rightarrow \infty)=$ $|\alpha\rangle\langle\alpha|$, as it should.

On the other hand, with a non-diagonal form of the Lindblad operators, cf (16), we would have contributions to $\dot{\rho}_{1,1}$ of the form $|\beta\rangle\langle\alpha|$ and its Hermitian adjoint in addition to the pure state contribution. These contributions clearly cannot be allowed, as $\rho_{1,1}$ should be independent of the state $\beta$ of the outgoing particle. Hence, we find that, up to an arbitrary phase factor, definition (17) is the proper way to define $A(x)$.

For a non-interacting $N$-fermion system where $N_{\alpha}$ particles are bound and $N_{\beta}$ particles are unbound, and with an initial state given by the Slater determinant $|\Psi\rangle=$ $\left|\left\{\alpha_{1}, \ldots, \alpha_{N_{\alpha}}, \beta_{1}, \ldots, \beta_{N_{\beta}}\right\}\right\rangle$, it may be verified by inspection that the source term for the $N_{\alpha}$ th block, $\rho_{N_{\alpha}, N_{\alpha}}$, is always proportional to $\left|\left\{\alpha_{1}, \ldots, \alpha_{N_{\alpha}}\right\}\right\rangle\left\langle\left\{\alpha_{1}, \ldots, \alpha_{N_{\alpha}}\right\}\right|$ given that none of the $\alpha_{i}$-states overlap with $\Gamma$. The intermediate density matrix blocks $\rho_{N-n, N-n}$, where $0<n<N_{\beta}$, will approach zero as $t \rightarrow \infty$, but transiently describe (mixed) states where $n$ particles have left.

Of course, in the more interesting context of interacting particles, the structure of the diagonal blocks of the density operator, $\rho_{n, n}$, is more complex than in the special case of non-interacting particles.

\subsection{Consequences and interpretation}

Typically, our starting point is a pure $N$-particle state, $|\Psi(0)\rangle\langle\Psi(0)|$, in which case (21) reduces to the ordinary Schrödinger equation (2), which was our original formulation. Moreover, it is easy to see that $\rho(t)$ will remain block diagonal for all $t$ in this case, i.e. $\rho_{n, m}=0$ if $n \neq m$. But
(21) shows that $\rho_{n, n}(t)$ in general is a mixed state, unlike $\rho_{N, N}(t)=|\Psi(t)\rangle\langle\Psi(t)|$. This is due to the information loss when admitting ignorance of the whereabouts of the removed particle.

The analogy to the situation described in relation to (8) is quite clear. With the possibility of spontaneous decay by emitting a photon, the equation dictating the evolution of $\rho^{(n)}$ acquires a source term depending on $\rho^{(n-1)}$, i.e. the atomic system with one less photon [16]. In this case, as in the cases of $[19,20]$, the Lindblad operators are related to the spontaneous decay rates.

We stress that by construction, $\operatorname{Tr}[\rho(t)]=1$ for all $t$. Probability flows monotonically from $\mathscr{H}_{N}$ into $\mathscr{H}_{n}$, and the particle absorbed from $\rho_{N, N}$ is not present in $\rho_{n, n}$, but is erased, and $\rho_{n, n}$ is a proper description of an $n$-fermion system. In this way, we see that the above construction is a natural generalization of the original non-Hermitian dynamics, which describes the classical removal of a particle, into one that also describes the remaining system in a consistent way. It is striking to note that the $\mathrm{CAP},-\mathrm{i} \Gamma(x)$, is already given as one of the terms in the Lindbladian, so that the Fock space formulation follows almost immediately.

It is worthwhile to mention that the traces of the blocks $\rho_{n, n}$ along the diagonal of $\rho$ have very simple interpretations as the probability $P(n)$ of having $n$ particles in the system upon a measurement, i.e.

$$
P(n) \equiv \operatorname{Tr}_{n}[\rho(t)] \equiv \operatorname{Tr}\left[\rho_{n, n}(t)\right] .
$$

In particular, $P(N)=\langle\Psi \mid \Psi\rangle \leqslant 1$, and $P(0)=1-$ $\sum_{n=1}^{N} P(n)$. Hence, within this formalism, distinguishing between single, double, etc ionization of atoms and molecules is straightforward.

For any observable $A$ the expectation value is given by $\langle A\rangle \equiv \operatorname{Tr}[A \rho]=\sum_{n} \operatorname{Tr}\left[A \rho_{n}\right]$. For example, the expected number of particles in the system is given by

$$
\langle\mathscr{N}\rangle=\int \mathrm{d} x \operatorname{Tr}\left[\psi^{\dagger}(x) \psi(x) \rho\right]=\sum_{n=1}^{N} n P(n) .
$$

It may also be useful to consider conditional expectation values:

$$
\langle A\rangle_{n} \equiv \frac{\operatorname{Tr}_{n}(A \rho)}{\operatorname{Tr}_{n}(\rho)},
$$

i.e. the expectation value of $A$ given that the system is found in an $n$-particle state.

Obviously, as particles are 'removed' by the absorber, information is lost. This information loss may be quantified by the von Neumann entropy, $S \equiv-\operatorname{Tr}[\rho \log \rho]$, or the closely related notion of purity, defined by [13]

$$
\varsigma \equiv \operatorname{Tr}\left(\rho^{2}\right) \leqslant 1
$$

Unity minus this quantity, $1-\varsigma$, is a measure of the amount of mixedness, and the purity $\varsigma$ is 1 for pure states only. Similar to conditional expectation values, cf (26), one may define conditional purity and von Neumann entropy as the corresponding quantity of the re-normalized block, i.e. $\rho_{n, n} / \operatorname{Tr}\left[\rho_{n, n}\right]$.

Of course, for a full quantum mechanical description in terms of the complete (unabsorbed) $\mathrm{N}$-particle wavefunction, 
no information is lost. Indeed, the absorption of particles is a semi-classical concept. In the full $\mathrm{N}$-fermion quantum system no such separation is possible. On the other hand, as the Schrödinger equation for the $N$ particles is separable in the non-interacting $\alpha_{i}$ and $\beta_{i}$ states discussed above, giving a Slater determinant of the time evolved one-particle states as the full solution, the Lindblad equation is seen to correctly construct the $N_{\alpha}$-particle Slater determinant resulting from the removal of the $N_{\beta}$ outgoing particles from this Slater determinant. Thus, the Lindblad equation exactly encapsulates the approximate separation of non-interacting quantum systems far apart.

\section{Example: two identical spin- $\frac{1}{2}$ particles in one dimension}

In the following we consider two fermions in one dimension with the one-body Hamiltonian $h$ given by

$$
h=-\frac{\hbar^{2}}{2 m} \frac{\partial^{2}}{\partial x^{2}}+V(x, t),
$$

where $V(x, t)$ is some external, possibly time-dependent, potential. The fermions interact via a potential $U\left(x_{1}-x_{2}\right)$ and the extension of the system is effectively reduced by imposing a CAP. With two interacting identical fermions, we are confined to the Hilbert space

$$
\mathscr{H}=\mathscr{H}_{2} \oplus \mathscr{H}_{1} \oplus \mathscr{H}_{0} .
$$

We discretize this system using a uniform grid in the interval $\left[0, x_{\max }\right)$ containing $N$ points $\left\{x_{j}\right\}_{j=0}^{N-1}, x_{j}=j h$, with $h=x_{\max } / N$. The field operators $\psi^{\dagger}(x)$ can be replaced by a finite number of creation operators $c_{j}^{\dagger}$, creating a particle at grid point $x_{j}$. These operators, which obey the usual fermion anti-commutator rules

$$
\left\{c_{j}, c_{k}\right\}=0, \quad\left\{c_{j}, c_{k}^{\dagger}\right\}=\delta_{j, k},
$$

map discrete anti-symmetrized $\delta$-function bases for $\mathscr{H}_{n}$ into bases for $\mathscr{H}_{n \pm 1}$.

The Hamiltonian takes the form

$$
H=\sum_{i, j} h_{i j} c_{i}^{\dagger} c_{j}+\frac{1}{2} \sum_{i, j} U\left(x_{i}-x_{j}\right) c_{i}^{\dagger} c_{j}^{\dagger} c_{j} c_{i},
$$

where $h_{i j}$ are the matrix elements of the one-body Hamiltonian, which depend on the chosen discretization of the second derivative. We choose a typical spectral approximation using the discrete Fourier transform, which also imposes periodic boundary conditions. The CAP is similarly discretized as

$$
-\mathrm{i} \Gamma=-\mathrm{i} \sum_{j} \Gamma\left(x_{j}\right) c_{j}^{\dagger} c_{j},
$$

where $\Gamma(x)$ is a non-negative function which increases as one approaches the boundary of the interval $\left[0, x_{\max }\right)$ and is zero in most of the interior.

Concerning the density operator $\rho$, we write $\rho=|\Psi\rangle\langle\Psi|+$ $\rho_{1}+\rho_{0}$, with $\rho_{n}=P_{n} \rho P_{n}$. Initially the system is prepared in a two-particle pure state localized inside the absorption-free part of the grid. The master equation for $\rho_{2}(t)$ is equivalent to the usual Schrödinger equation (2) for $|\Psi(t)\rangle$, while the master equation for $\rho_{1}(t)$ acquires a source term, i.e.

$$
\begin{gathered}
\mathrm{i} \hbar \frac{\mathrm{d}}{\mathrm{d} t} \rho_{1}(t)=\left[H, \rho_{1}(t)\right]-\mathrm{i}\left\{\Gamma, \rho_{1}(t)\right\} \\
\quad+2 \mathrm{i} \sum_{j} \Gamma\left(x_{j}\right) c_{j}|\Psi(t)\rangle\langle\Psi(t)| c_{j}^{\dagger} .
\end{gathered}
$$

It is natural to view the discrete one-particle wavefunction as a vector $\psi_{1}\left(x_{j}\right)$ of length equal to the number of discrete degrees of freedom. Similarly, it is natural to view twoparticle wavefunctions as anti-symmetric matrices $\psi_{2}\left(x_{j}, x_{k}\right)$. Moreover, a one-particle density matrix becomes a Hermitian matrix $\rho_{1}\left(x, x^{\prime}\right)$. Using these notions, the master equation (33) can be compactly written as

$$
\begin{gathered}
\mathrm{i} \hbar \frac{\mathrm{d}}{\mathrm{d} t} \rho_{1}(t)=\left[H, \rho_{1}(t)\right]-\left\{\Gamma, \rho_{1}(t)\right\}+2 \mathrm{i} \rho_{S} \\
\text { with } \quad \rho_{S} \equiv 2 h \psi_{2}^{\dagger} \Gamma \psi_{2},
\end{gathered}
$$

where the last term contains matrix-matrix products. The extra factor 2 in $\rho_{S}$ originates from the fact that the matrix $\psi_{2}$ relates to a (redundant) 'basis' of direct product states.

If the initial two-particle state is an eigenstate of the total spin and its projection, the source term is always proportional to a single one-particle spin state. Hence, the spin does not introduce any complication in the notation in this case. For parallel spins, the one-particle spin has the same direction as the two-particle spins, and for spin projection $M_{S}=0$ the one-particle density operator has its spinor component given by $\left(|\uparrow\rangle+(-1)^{S}|\downarrow\rangle\right) / \sqrt{2}$, where $S$ is the total spin eigenvalue.

The equation for $\rho_{0}=p_{0}(t)|-\rangle\langle-|$ becomes

$$
\frac{\mathrm{d}}{\mathrm{d} t} p_{0}(t)=\frac{2}{\hbar} \sum_{j} \Gamma\left(x_{j}\right) c_{j} \rho_{1} c_{j}^{\dagger} .
$$

In principle, it is not necessary to include this equation in our calculations, as $p_{0}(t)$ can be calculated from the constraint $\operatorname{Tr}[\rho(t)]=1$.

\subsection{Collision in a Gaussian well}

We will now focus on an example in which we set $\hbar=m=1$ and place the electrons in a potential of Gaussian form:

$$
V(x)=-V_{0} \exp \left(-\frac{\left(x-x_{0}\right)^{2}}{2 \sigma^{2}}\right) .
$$

The particles interact via a regularized Coulomb interaction

$$
U\left(\left|x_{1}-x_{2}\right|\right)=\frac{\lambda}{\sqrt{\left(x_{1}-x_{2}\right)^{2}+\delta_{C}^{2}}} .
$$

For this problem we choose a CAP of power form:

$$
\begin{aligned}
& \Gamma(x)=C\left(\frac{\xi}{x_{T}}\right)^{n}, \\
& \xi \equiv \max \left\{0, x_{T}-x, x-\left(x_{\max }-x_{T}\right)\right\},
\end{aligned}
$$

where $n \geqslant 1$ and $x_{T}$ is the distance from the edges at which the CAP is 'turned on', see figure 2.

The system may for instance serve as a model for a quantum dot which couples to the conduction band and has narrow confinement in two dimensions. 


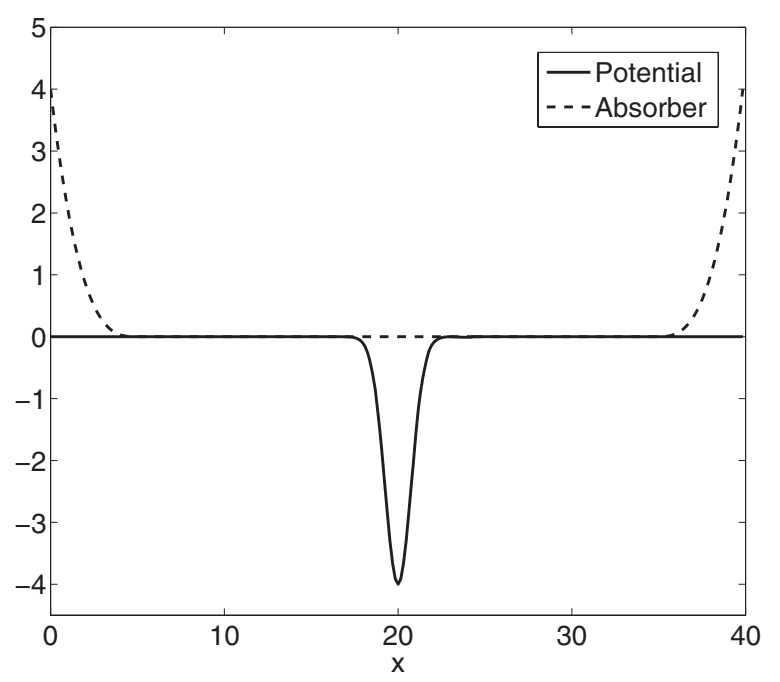

Figure 2. The Gaussian well $V(x), \operatorname{cf}(36)$, and the absorber $\Gamma(x)$, $\mathrm{cf}(38)$. The well has a depth of 4 and a width of 0.75 , and the absorber has a third-order power form for distances less than 5 from the edges at $x=0$ and $x=40$.

Equation (34) is integrated using a scheme of second order in the time step based on a standard split-step operator scheme [21]. It is instructive to consider a more general setting in order to introduce the time-stepping scheme for the density matrix. Given a differential equation for an entity $y(t)$ in a linear space of the form

$$
\dot{y}(t)=L_{t} y(t)+f(t),
$$

where $L_{t}$ is a linear operator dependent on $t$ and $f(t)$ is a source term independent of $y(t)$, we may integrate formally using standard time-ordering techniques to obtain

$$
\begin{aligned}
y(t) & =\mathscr{T} \mathrm{e}^{\int_{0}^{t} L_{s} \mathrm{~d} s} y(0)+F(t) \\
& +\sum_{n=1}^{\infty} \underbrace{\int_{0}^{t} \cdots \int_{0}^{s_{n-1}}}_{n \text {-fold }} L_{s_{1}} \cdots L_{s_{n}} F\left(s_{n}\right) \mathrm{d} s_{n} \cdots \mathrm{d} s_{1},
\end{aligned}
$$

with $F(t)=\int_{0}^{t} f(s) \mathrm{d} s$. The source terms are not easily transformed using the time-ordering operator $\mathscr{T}$. Assuming that the case $f(t)=0$ can be integrated to $p$ th order in the time $t$ using $y(t)=\mathscr{U}_{t} y(0)$, a $p$ th order method for the general case can be obtained by keeping $p$ source terms and evaluating these with sufficiently high-order quadrature. For example, using standard Strang splitting which gives a scheme of local error $O\left(t^{3}\right)$, or other schemes based on the Magnus expansion [22], we may approximate the term $F(t)$ as $F(t) \approx t[f(0)+f(t)] / 2$ (using trapezoidal quadrature) and the $n=1$ term as $\int_{0}^{t} L_{s} F(s) \mathrm{d} s \approx t^{2} L_{t / 2}[f(0)+t \dot{f}(0) / 2]$. Specifically, in our implementation we have used a secondorder scheme given by

$$
\begin{aligned}
\rho_{1}(t)= & \mathrm{e}^{-\mathrm{i}(V+U-\mathrm{i} \Gamma) t / 2} \mathrm{e}^{-\mathrm{i} T t} \mathrm{e}^{-\mathrm{i}(V+U-\mathrm{i} \Gamma) t / 2} \rho_{1}(0) \\
& \times \mathrm{e}^{+\mathrm{i}(V+U+\mathrm{i} \Gamma) t / 2} \mathrm{e}^{-\mathrm{i} T t} \mathrm{e}^{+\mathrm{i}(V+U+\mathrm{i} \Gamma) t / 2}+2 t \rho_{S}(0) \\
& +t^{2}\left[\dot{\rho}_{S}(0)-\mathrm{i}\left(H \rho_{S}(0)-\rho_{S}(0) H^{\dagger}\right)\right]+O\left(t^{3}\right) \\
& \text { where } \quad \dot{\rho}_{S}=2 h\left(\dot{\psi}_{2} \Gamma \psi_{2}^{\dagger}+\psi_{2} \Gamma \dot{\psi}_{2}^{\dagger}\right) .
\end{aligned}
$$

Here, $T$ is the kinetic energy. As this scheme is trace preserving to second order only, we have also solved (35) in order to check that our numerical time step is small enough to preserve the total trace.

Figure 3 shows the evolution of the particle density for a system in which a fermion collides with another one. In this case the potential depth $V_{0}=4$ and the width $\sigma=0.75$, the interaction strength $\lambda=5$ and the 'softening' $\delta_{C}=0.1$. The CAP has the power $n=3$, the strength $C=4$ and $x_{T}=5$. The starting point is a spatially anti-symmetrized state (spin triplet) consisting of a particle trapped in the well and an incoming wave packet of Gaussian shape. The trapped part corresponds to a superposition of the ground and the first excited one-particle states in the well, and the incoming wavefunction has mean momentum $k_{0}=2$. It is seen that as absorption, both due to transmission and back-scattering, takes place, the two-particle density vanishes and a oneparticle density emerges. It is also seen that, apart from in the absorption region, the total particle density, obtained by adding the two- and one-particle densities, compares well to the 'true' particle density obtained from solving the Schrödinger equation without absorber on a larger grid.

Due to the collision, there is a finite probability that both particles are absorbed. This is clearly seen in figure 4, which shows how the total trace is distributed between the sub-spaces $\mathscr{H}_{2}, \mathscr{H}_{1}$ and $\mathscr{H}_{0}$ as a function of time. In this particular case we have $P(1 ; t \rightarrow \infty)=0.92$ and $P(0 ; t \rightarrow \infty)=0.077$. Also, shown are the expectation value of the particle number and the purity of the system. Purity is reduced in two ways. Firstly, it is reduced as the trace becomes distributed between the three sub-systems, and secondly because $\rho_{1}$ is not a pure state within $\mathscr{H}_{1}$. This is seen from the fact that conditional purity, $\varsigma_{1}$, converges towards 0.6 , i.e. less than unity (not shown explicitly in the figure).

\subsection{Laser ionization of a one-dimensional helium atom}

In the next example we expose our system to an electric pulse of type

$$
E(t)=E_{0} \sin ^{2}\left(\frac{\pi t}{T}\right) \cos (\omega t) .
$$

With this time-dependent perturbation, the one-particle Hamiltonian (28) acquires a time-dependent term, which in the length gauge representation reads

$$
x E(t) .
$$

We have here set the charge of the particles (the electrons) to be -1 . The static potential $V_{N}$ felt by the particles is chosen to be a regularized Coulomb potential:

$$
V_{N}(x)=-\frac{2}{\sqrt{x^{2}+\delta_{N}^{2}}},
$$

whereas the interaction between them is still described by (37). By choosing $\delta_{N}^{2}=\frac{1}{2}$ au and $\delta_{C}=0.5735$ au, the ground state energy and the first ionization threshold coincide with those of a true three-dimensional atom, i.e. the ground state energy is $-2.904 \mathrm{au}$ and the ground state energy of ' $\mathrm{He}^{+}$' is $-2 \mathrm{au}$. By 'au' we mean atomic units, defined by choosing the Bohr radius, the electron mass, the elementary charge and $\hbar$ as units for their respective quantities. The ground state of the system, 

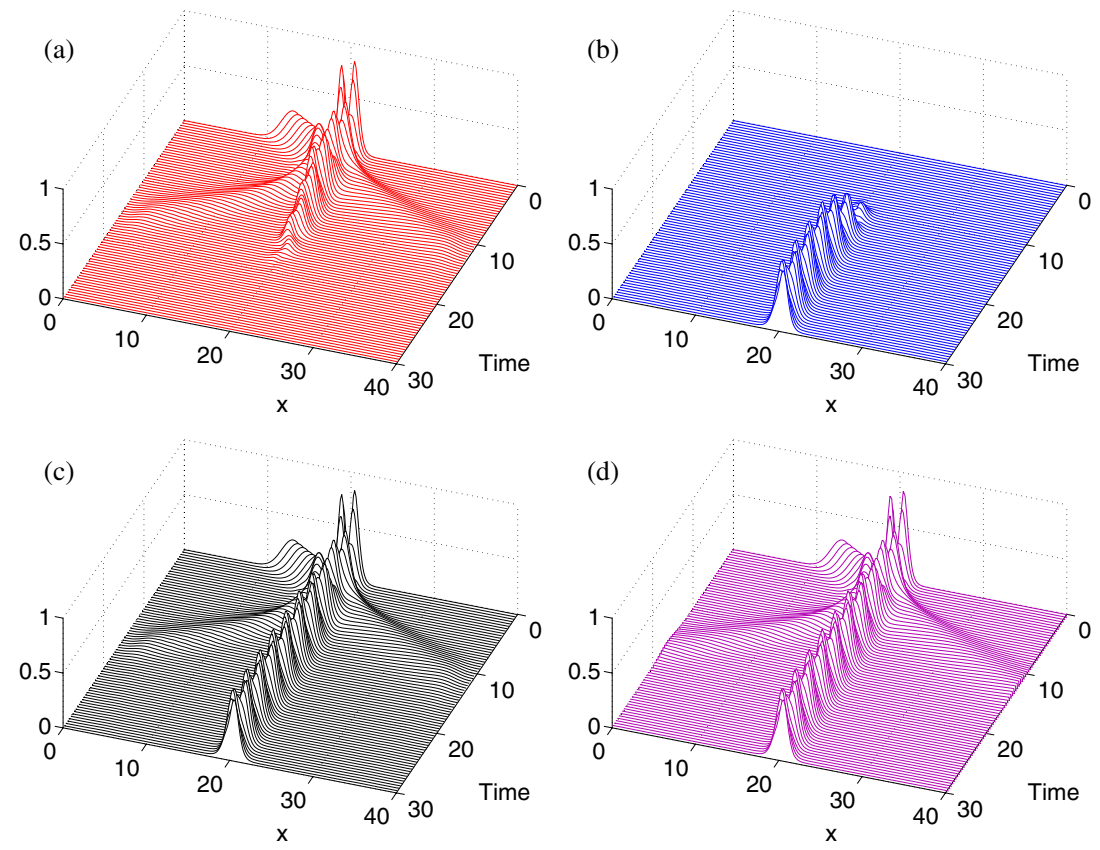

Figure 3. The evolution of the particle density. Panel (a) is the particle density within the two-particle system, panel (b) is obtained from the one-particle density operator and panel (c) is the total particle density. Also shown, panel (d), is the particle density in the same region obtained from a solution of the full two-particle problem on a larger grid without absorber. The initial state is an anti-symmetrized state consisting of a Gaussian wave travelling towards the right with mean momentum $k_{0}=2$ and a state corresponding to a particle initially trapped in the well.
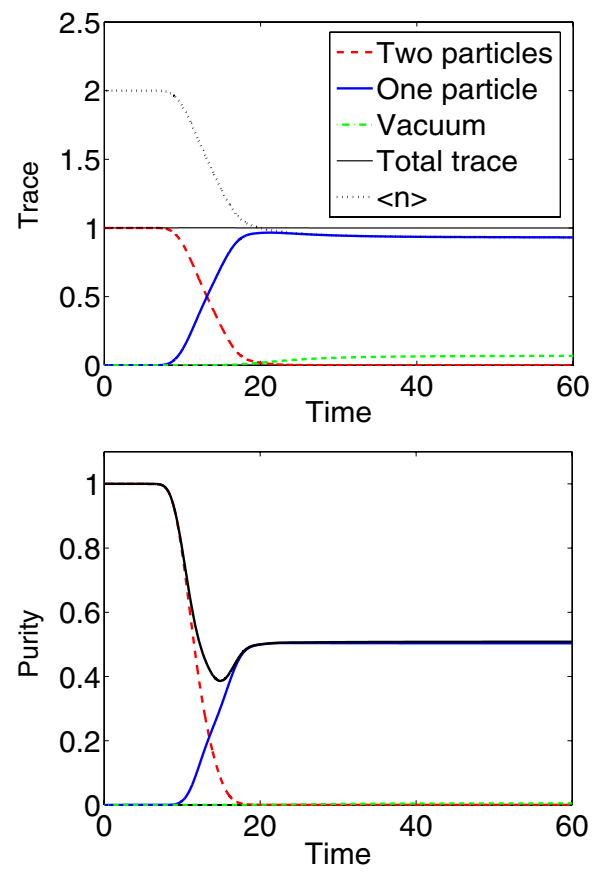

Figure 4. The trace and the expectation value of the number operator (upper panel) and the purity of the system (lower panel). In both cases the separate contributions from the two, one and zero part of the total density operator have been shown.

which is a spin singlet state, is easily obtained by propagation in imaginary time.

Figure 5 shows the evolution of the system exposed to a pulse of maximum field strength $E_{0}=5$ au, central frequency $\omega=3.2 \mathrm{au}$ and a duration corresponding to three optical cycles. This central frequency corresponds to a photon energy which energetically allows for one-photon double ionization. Along with a figure showing how the partial traces, given by (24), evolve in time, and another one showing the time dependence of the electric field, given by (42), we have included snapshots of the absolute values of the two-particle wavefunction $\psi_{2}\left(x_{1}, x_{2}\right)$ and the one-particle density matrix $\rho_{1}\left(x, x^{\prime}\right)$. It is clearly seen that as absorption takes place in the two-particle sub-system, a one-particle density matrix emerges. Note that the lobes following the axes of the $\left(x, x^{\prime}\right)$ coordinate system do not correspond to absorption of the second electron but rather to loss of coherence within the one-particle sub-system. However, from the lower panel of figure 5 , which clearly demonstrates how single ionization may be distinguished from double ionization, we see that there is a finite probability of absorbing both particles. In this case, specifically, the probability of ionizing only one electron is $P(1 ; t \rightarrow \infty)=0.31$, and the probability of double ionization is $P(0 ; t \rightarrow \infty)=0.034$.

In both the examples we see that the systems, with a certain probability, undergo incoherent transitions from a two-particle system $(\mathrm{He})$ to a one-particle system $\left(\mathrm{He}^{+}\right)$and a zero-particle system $\left(\mathrm{He}^{2+}\right)$. A similar mechanism is seen in the work by $\mathrm{H}$ Leth et al [23], which describes double photo-ionization of the hydrogen molecule using the Monte Carlo wave packet method [24]. Here transitions from the $\mathrm{H}_{2}$ system to $\mathrm{H}_{2}^{+}$and $\mathrm{H}_{2}^{2+}$ are imposed by continuous gedanken measurements on the photo-electrons. Their results compare very well with experiment. 
(a)

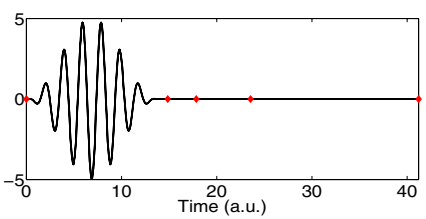

(c)

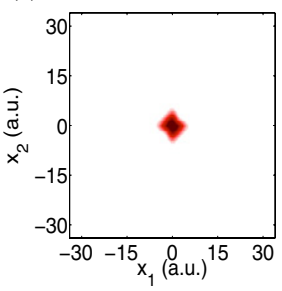

(d)

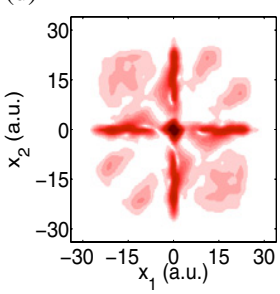

(e)

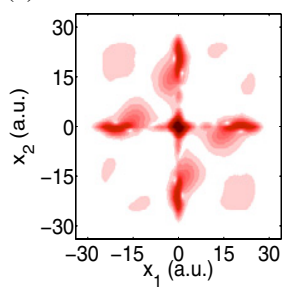

(i)

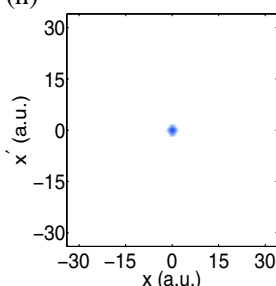

(b)

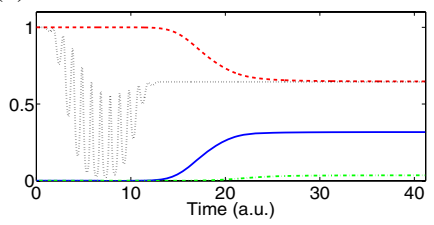

(f)

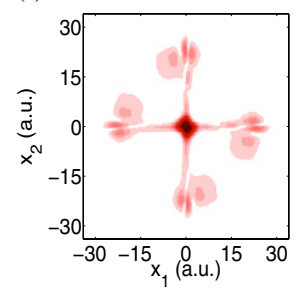

(g)

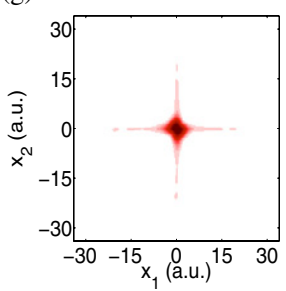

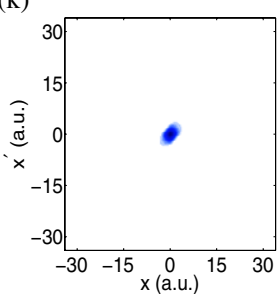

(k)
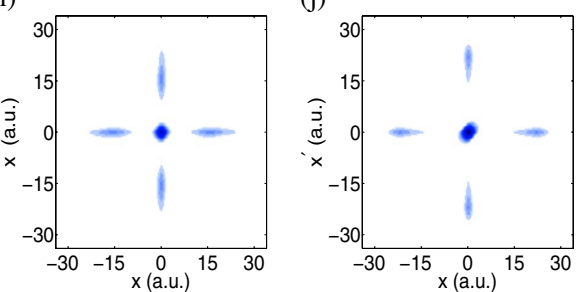

Figure 5. The panels show the evolution of a model one-dimensional helium atom exposed to a five-cycle sine-squared electromagnetic pulse of strength $E_{0}=5 \mathrm{au}$ and central frequency $\omega=3.2 \mathrm{au}$ (panel (a)). Panel (b) shows the probability of finding two (dashed curve), one (full curve) and zero particles (dash-dotted curve) in the system in the same manner as in the upper panel of figure 4 . The dotted curve shows the probability of the system being in the initial state, $\left|\left\langle\psi_{2}(t=0) \mid \psi_{2}(t)\right\rangle\right|^{2}$. The middle row, panels (c)- $(\mathrm{g})$, shows the absolute value of the wavefunction of the two-particle part $\psi_{2}\left(x_{1}, x_{2}\right)$ at various instances during the evolution. The instances of each 'snapshot' is indicated by diamonds in panel (a). The lower row, panels (h)-(k), shows the absolute value of the one-particle part of the density matrix $\rho_{1}\left(x, x^{\prime}\right)$ at the same instances (except at $\left.t=0\right)$. The same colour scaling has been used in all panels $((\mathrm{c})-(\mathrm{k}))$.

\section{Conclusion}

In conclusion, we have demonstrated how the Lindblad equation in Fock space can be used to generalize the notion of absorbing boundary conditions for $\mathrm{N}$-particle systems. Specifically, the remainder of the system is preserved as a particle is absorbed. With this formalism it may be possible to describe the dynamics of unbound systems which otherwise would require an unrealistically large numerical grid.

As a consequence of this being a Markovian process, some coherence is lost, and the state after absorption is in general given by a mixed state rather than a wavefunction. Within Lindblad theory, the identification between the Lindblad operators and the creation and annihilation operators comes quite natural for complex absorbing potentials. It should be possible to extend the theory outlined here also to other kinds of formalisms involving non-Hermitian Hamiltonians, such as complex scaling. This may necessitate a modification of the underlying inner product.

Since it is considerably more involved to solve a master equation rather than a Schrödinger equation, cf [23, 24], future work will aim to find lower rank methods for solving (21). Also, more sophisticated spatial approximations like sparse grids [22] may be utilized to simplify the treatment of more particles.

\section{Acknowledgments}

Constructive suggestions from Michael Genkin and from the referees are gratefully acknowledged by the authors.

\section{References}

[1] Parker J, Taylor K T, Clark C W and Blodgett-Ford S 1996 J. Phys. B: At. Mol. Opt. Phys. 29 L33

[2] Uiberacker M et al 2007 Nature 446627

[3] Kosloff R and Kosloff D 1986 J. Comput. Phys. 63 363-76

[4] Fevens T and Jiang H 1999 SIAM J. Sci. Comput. 21 255-82

[5] Antoine X, Arnold A, Besse C, Ehrhardt M and Schadle A 2008 Commun. Comput. Phys. 4 729-96

[6] Berenger J 1994 J. Comput. Phys. 114 185-200

[7] Manolopoulos D E 2002 J. Chem. Phys. 117 9552-9

[8] Monnerville M and Robbe J 1999 Eur. Phys. J. D 5 381-7

[9] Grozdanov T, Andric L and McCarroll R 2006 J. Chem. Phys. 124094303

[10] Karlsson H O 2009 J. Phys. B: At. Mol. Opt. Phys. 42125205

[11] Kulander K C 1987 Phys. Rev. A 35 445-7

[12] Protopapas M, Keitel C and Knight P 1996 J. Phys. B: At. Mol. Opt. Phys. 29 L591-8

[13] Schlosshauer M 2007 Decoherence and the Quantum-to-Classical Transition (Berlin: Springer)

[14] Sandulescu A and Scutaru H 1987 Ann. Phys. 173 277-317

[15] Plenio M B and Knight P L 1998 Rev. Mod. Phys. 70 101-44

[16] Mollow B R 1975 Phys. Rev. A 12 1919-43

[17] Gorini V, Kossakowski A and Sudarshan E 1976 J. Math. Phys. 17 821-5 
[18] Lindblad G 1976 Commun. Math. Phys. 48119

[19] Caban P, Rembieliński J, Smoliński K A and Walczak Z 2005 Phys. Rev. A 72032106

[20] Bertlmann R A, Grimus W and Hiesmayr B C 2006 Phys. Rev. A 73054101

[21] Feit M, Fleck J Jr and Steiger A 1982 J. Comput. Phys. $47412-33$
[22] Lubich C 2008 From Quantum to Classical Molecular Dynamics: Reduced Models and Numerical Analysis (Zürich: European Mathematical Society)

[23] Leth H A, Madsen L B and Mølmer K 2009 Phys. Rev. Lett. 103183601

[24] Dalibard J, Castin Y and Mølmer K 1992 Phys. Rev. Lett. $68580-3$ 